NTERnational Electronic Journal of Algebra

Volume 22 (2017) 78-96

DOI: $10.24330 /$ ieja.325927

\title{
ON THE NON-NILPOTENT GRAPHS OF A GROUP
}

\author{
Deiborlang Nongsiang and Promode Kumar Saikia \\ Received: 10 November 2016; Revised: 17 April 2017 \\ Communicated by Burcu Üngör
}

\begin{abstract}
Let $G$ be a group and $\operatorname{nil}(G)=\{x \in G \mid\langle x, y\rangle$ is nilpotent for all $y \in G\}$. Associate a graph $\mathfrak{R}_{G}$ (called the non-nilpotent graph of $G$ ) with $G$ as follows: Take $G \backslash \operatorname{nil}(G)$ as the vertex set and two vertices are adjacent if they generate a non-nilpotent subgroup. In this paper we study the graph theoretical properties of $\mathfrak{R}_{G}$. We conjecture that the domination number of the non-nilpotent graph of every finite non-abelian simple group is 2 . We also conjecture that if $G$ and $H$ are two non-nilpotent finite groups such that $\mathfrak{R}_{G} \cong \mathfrak{R}_{H}$, then $|G|=|H|$. Among other results, we show that the nonnilpotent graph of $D_{10}$ is double-toroidal.
\end{abstract}

Mathematics Subject Classification (2010): 20D60

Keywords: Non-nilpotent graph, finite group

\section{Introduction}

Let $G$ be a group and $\operatorname{nil}(G)=\{x \in G \mid\langle x, y\rangle$ is nilpotent for all $y \in G\}$. Associate a simple graph $\mathfrak{R}_{G}$ (called the non-nilpotent graph) with $G$ as follows: the vertex set $V\left(\Re_{G}\right)$ is $G \backslash \operatorname{nil}(G)$ and the edge set $E\left(\Re_{G}\right)$ consists of $\{x, y\}$, where $x$ and $y$ are distinct elements of $G \backslash \operatorname{nil}(G)$ such that $\langle x, y\rangle$ is not nilpotent. It is not known whether the subset $\operatorname{nil}(G)$ is a subgroup of $G$, but in many important cases it is a subgroup. In particular, $\operatorname{nil}(G)$ is equal to the hypercenter $Z^{*}(G)$ of $G$, whenever $G$ satisfies the maximal condition on its subgroups or $G$ is a finitely generated solvable group (see [2, Proposition 2.1]). The non-nilpotent graph may be regarded as generalization of the non-commuting graph considered in [1,3]. Recently, in [2], some group and graph properties of the non-nilpotent graph associated to a group are studied.

In Section 3 of the paper, we study some properties of the non-nilpotent graph $\mathfrak{R}_{G}$ of a non-weakly nilpotent group $G$ (a group is said to be weakly nilpotent if every two generated subgroup of $G$ is nilpotent). We completely characterize periodic groups with $\operatorname{nil}(G)$ a subgroup whose non-nilpotent graph have domination

The first author wishes to express his sincere thanks to CSIR(India) for its financial assistance (File No. 09/347(0209)/2012-EMR-I). 
number 1 . We see that for a finite group $G, \mathfrak{R}_{G}$ is a complete multi-partite graph if and only if $G$ is an $\mathfrak{n n}$-group. We also give an example of a finite group whose non-nilpotent graph has diameter 3 .

In Section 4, we study groups with isomorphic non-nilpotent graph. We show that the non-nilpotent graph of a finite group cannot be isomorphic to the nonnilpotent graph of an infinite one. We also concentrate on the following conjecture:

Conjecture 1.1. Let $G$ and $H$ be two non-nilpotent finite groups such that $\mathfrak{R}_{G} \cong$ $\Re_{H}$. Then $|G|=|H|$.

The above conjecture is proved when one of the groups in the conjecture is $D_{2 n}$ ( $n$ not a power of 2$), P S L(2, q)\left(q^{2} \not \equiv 1 \bmod 16\right)$, the group of order $p q$ or when one of the groups has clique number 4 .

In Section 5, we study the genus of the non-nilpotent graph $\mathfrak{R}_{G}$ of a non-weakly nilpotent group $G$. We show that the hypercenter of $G$ is bounded by a function of the genus of $\mathfrak{R}_{G}$. We also shown that, for any finite group $G, \mathfrak{R}_{G}$ is not toroidal.

In Section 6, we find clique and chromatic number of some groups.

\section{Some prerequisites}

In this section, we recall certain group theoretic and graph theoretic terminologies along with some well-known results which are used in the forthcoming sections.

An ascending series $1=G_{0} \triangleleft G_{1} \triangleleft \ldots G_{\beta}=G$ in a group $G$ is said to be central if $G_{\alpha} \triangleleft G$ and $G_{\alpha+1} / G_{\alpha}$ lies in the center of $G / G_{\alpha}$ for every $\alpha<\beta$. A group which possesses a central ascending series is called hypercentral. If $G$ is any group and $\alpha$ an ordinal, the terms $Z_{\alpha}(G)$ of the upper central series of $G$ are defined by the rules

$$
Z_{0}(G)=\{1\} \text { and } Z_{\alpha+1}(G) / Z_{\alpha}(G)=Z\left(G / Z_{\alpha}(G)\right)
$$

together with the completeness condition

$$
Z_{\lambda}(G)=\bigcup_{\alpha<\lambda} Z_{\alpha}(G)
$$

where $\lambda$ is a limit ordinal. Since the cardinality of $G$ cannot be exceeded, there is an ordinal $\beta$ such that $Z_{\beta}(G)=Z_{\beta+1}(G)$, etc., a terminal subgroup called the hypercenter of $G$ and is denoted by $Z^{*}(G)$.

A group $G$ is said to be an $A C$-group if the centralizer $C_{G}(x)$ of every non-central element $x$ in $G$ is an abelian subgroup of $G$. The $A C$-groups have been extensively studied by many authors (see, for example, [1], [14], [15]). 
The vertex set and edge set of a graph $\Gamma$ is denoted by $V(\Gamma)$ and $E(\Gamma)$, respectively. A subset of the vertex set of a graph $\Gamma$ is called a clique of $\Gamma$ if it consists entirely of pairwise adjacent vertices. The least upper bound of the sizes of all the cliques of $G$ is called the clique number of $\Gamma$, and is denoted by $\omega(\Gamma)$. The chromatic number of a graph $\Gamma$, written $\chi(\Gamma)$, is the minimum number of colors needed to label the vertices so that adjacent vertices receive different colors. Clearly, $\omega(\Gamma) \leq \chi(\Gamma)$. For a graph $\Gamma$ and a subset $S$ of the vertex set $V(\Gamma)$, denote by $N_{\Gamma}[S]$ the set of vertices in $\Gamma$ which are in $S$ or adjacent to a vertex in $S$. If $N_{\Gamma}[S]=V(\Gamma)$, then $S$ is said to be a dominating set (of vertices of $V(\Gamma)$ ). The domination number of a graph $V(\Gamma)$, denoted by $\lambda(V(\Gamma))$, is the minimum size of a dominating set of the vertices in $V(\Gamma)$. A subset $X$ of the vertices of $\Gamma$ is called an independent set if the induced subgraph on $X$ has no edges. The maximum size of an independent set in a graph $\Gamma$ is called the independent number of $\Gamma$ and denoted by $\alpha(\Gamma)$. If a graph $\Gamma$ is connected, then the largest distance between all pairs of the vertices of $\Gamma$ is called the diameter of $\Gamma$ and it is denoted by $\operatorname{diam}(\Gamma)$. A complete multi-partite graph is the one whose vertex set can be partitioned into $m$ disjoint parts in such a way that two vertices are adjacent if and only if they lie in different parts. The complete multi-partite graph, with parts of size $n_{1}, \ldots, n_{m}$, is denoted by $K_{n_{1}, \ldots, n_{m}}$.

Let $U$ be a nonempty subset of the vertex set of a graph $\Gamma$. The induced subgraph of $\Gamma$ on $U$ is defined to be the graph $\Gamma[U]$ in which the vertex set is $U$ and the edge set consists precisely of those edges in $\Gamma$ whose endpoints lie in $U$.

The vertex connectivity, $\kappa(\Gamma)$, of a connected graph $\Gamma$ is the smallest number of vertices whose removal disconnect $\Gamma$. A subset $S$ of the vertices of a connected graph $\Gamma$ is called a cut set, if $\Gamma \backslash S$ is not a connected graph.

The genus of a graph $\Gamma$, denoted by $\gamma(\Gamma)$, is the smallest non-negative integer $g$ such that the graph can be embedded on the surface obtained by attaching $g$ handles to a sphere. Clearly, if $\tilde{\Gamma}$ is a subgraph of $\Gamma$, then $\gamma(\tilde{\Gamma}) \leq \gamma(\Gamma)$. Graphs having genus zero are called planar graphs, while those having genus one are called toroidal graphs. Graphs having genus two are called double-toroidal graphs. If $n \geq 3$, then it is well-known (see [19, Theorem 6-38]) that the genus of the complete graph $K_{n}$ is given by

$$
\gamma\left(K_{n}\right)=\left\lceil\frac{(n-3)(n-4)}{12}\right\rceil .
$$

On the other hand, if $m, n \geq 2$, then it is also well-known (see [19, Theorem 6-37]) that the genus of the complete bipartite graph $K_{m, n}$ is given by

$$
\gamma\left(K_{m, n}\right)=\left\lceil\frac{(m-2)(n-2)}{4}\right\rceil \text {. }
$$




\section{Some properties of non-nilpotent graphs}

Let $G$ be a group and $x \in G$. Then,

$$
\operatorname{nil}_{G}(x)=\{g \in G \mid\langle g, x\rangle \text { is nilpotent }\} .
$$

We call $\operatorname{nil}_{G}(x)$ the nilpotentizer of $x$ in $G$. For a nonempty subset of $G$, the nilpotentizer of $S$ in $G$ is defined as

$$
\operatorname{nil}_{G}(S)=\bigcap_{x \in S} \operatorname{nil}_{G}(x)
$$

We call $n i l_{G}(G)$ the nilpotentizer of $G$, and it will be denoted by $\operatorname{nil}(G)$. Thus $\operatorname{nil}(G)=\{x \in G \mid\langle x, y\rangle$ is nilpotent of all $y \in G\}$. Following [6], a group $G$ is call an $\mathfrak{n n}$-group if $\operatorname{nil}_{G}(x)$ is a nilpotent subgroup of $G$ for all $x \in G \backslash \operatorname{nil}(G)$.

We begin the section with the following result which enables us, in particular, to use $Z^{*}(G)$ and $\operatorname{nil}(G)$ interchangeably whenever the group $G$ satisfies the maximal condition on its subgroups or $G$ is a finitely generated solvable group.

Proposition 3.1. [2, Proposition 2.1] Let $G$ be a group. Then:

(1) $Z^{*}(G) \subseteq \operatorname{nil}(G) \subseteq R(G)$, where $R(G)$ is the set of right Engel elements of $G$.

(2) If $G$ satisfies the maximal condition on its subgroups or $G$ is finitely generated solvable group, then $Z^{*}(G)=\operatorname{nil}(G)=R(G)$.

Before stating the propositions that follow, it might be worth observing that $G \neq \operatorname{nil}(G)$ if and only if $G$ is not weakly nilpotent.

Proposition 3.2. Let $G$ be a non-weakly nilpotent group.

(1) If nil $(G)$ is a subgroup and $\{x\}$ is a dominating set for $\mathfrak{R}_{G}$, then $\operatorname{nil}(G)=$ $1, x^{2}=1$ and $\operatorname{nil}_{G}(x)=\langle x\rangle$.

(2) If $\operatorname{nil}(G)$ is a subgroup and $G$ is periodic, then $\lambda(G)=1$ if and only if $G$ contains a normal abelian subgroup $A$ with no element of order 2 and an element $x$ of order 2 such that $a^{x}=a^{-1}$ for all $a \in A$ and $G=A\langle x\rangle$ and $A \cap\langle x\rangle=1$.

Proof. (1) If $\operatorname{nil}(G)$ contains a non-trivial element $z$, then, since $\operatorname{nil}(G)$ is a subgroup of $G$, we have $x z \notin n i l(G)$, that is $x z \in V\left(\Re_{G}\right)$. Clearly, $x z$ is not adjacent to $x$, a contradiction, so $\operatorname{nil}(G)=1$. Also if $x^{2} \neq 1$, then $x^{-1}$ is not adjacent to $x$. Now since $\operatorname{nil}(G)=1$ and $x$ is adjacent to all vertices of $\Re_{G}, n i l_{G}(x)=\langle x\rangle$.

(2) Suppose that $\lambda\left(\mathfrak{R}_{G}\right)=1$. Thus $G$ contains a non-identity element $x$ such that $\{x\}$ is a dominating set for $\mathfrak{R}_{G}$. By part (1) we have $n i l_{G}(x)=\langle x\rangle$ and 
$x^{2}=1$. It follows that $\langle x\rangle \cap\left\langle x^{g}\right\rangle=1$ for all $g \in G \backslash\langle x\rangle$. Now by [17, Theorem 5], $A=G \backslash\left\{x^{g} \mid g \in G\right\}$ is a normal abelian subgroup of $G$, such that $G=A\langle x\rangle$ and obviously $A \cap\langle x\rangle=1$. Thus $G$ is a solvable periodic group which implies that $G$ is locally finite. Let $a \in A$ be a non-trivial element of $A$, then $B=\left\langle a, a^{x}\right\rangle$ is a finite abelian normal subgroup of $G$. Hence $x$ induces a fixed-point-free automorphism of order 2 in $B$, which implies that $B$ is an abelian group of odd order (see, for example, [13, Exercise 10.5.1]) and also we have $b^{x}=b^{-1}$ for all $b \in B$.

Now assume that $G$ contains a normal abelian subgroup $A$ and an element $x$ of order 2 with the properties stated in the proposition. It is easy to see that $\operatorname{nil}(G)=1$ and the subgroup generated by any elements of $G \backslash\langle x\rangle$ and $x$ is not nilpotent, so $\{x\}$ is a dominating set.

Question 3.3. Is the hypothesis "nil $(G) \leq G$ " necessary in Proposition 3.2?

Remark 3.4. Let $G$ be a group which is not weakly nilpotent. Then a subset $S$ of $V\left(\Re_{G}\right)$ is a dominating set if and only if $n_{i} l_{G}(S) \subset \operatorname{nil}(G) \cup S$. To see this, suppose $S$ is a dominating set. If a $\notin \operatorname{nil}(G) \cup S$, then by definition of dominating set, there exists $x \in S$, such that $\langle x, a\rangle$ is not nilpotent. Thus $a \notin n i l_{G}(S)$. It follows that $\operatorname{nil}_{G}(S) \subset S \cup \operatorname{nil}(G)$.

Now assume that nil $\operatorname{na}_{G}(S) \subset \operatorname{nil}(G) \cup S$. If a $\notin \operatorname{nil}(G) \cup S$, then by hypothesis, $a \notin n i l_{G}(S)$. Therefore, $a$ is adjacent to at least one element of $S$. This completes the proof.

Remark 3.5. If $G$ is a non-weakly nilpotent $\mathfrak{n n}$-group, then for all $x, y \in G \backslash \operatorname{nil}(G)$ with $\langle x, y\rangle$ not nilpotent, we have $\{x, y\}$ is a domination set for $\mathfrak{R}_{G}$. To see this, we need only to note that if $a, b, c \in G \backslash \operatorname{nil}(G)$, such that $\langle a, b\rangle$ and $\langle b, c\rangle$ are nilpotent, then $\langle a, c\rangle$ is nilpotent. Thus domination number of $\mathfrak{R}_{G}$ is less than or equal to 2.

Proposition 3.6. The domination number of the non-nilpotent graph of $A_{5}$ is 2 .

Proof. First note that $A_{5}$ is an $\mathfrak{n n}$-group. Thus, by the above remark, domination number of $\mathfrak{R}_{A_{5}}$ is less than or equal to 2 . Since $A_{5}$ is a simple group, $A_{5}$ does not contain a non-trivial proper normal subgroup. Thus Proposition 3.2 completes the proof.

We need the following result in the sequel.

Proposition 3.7. Let $G=S z\left(2^{2 m+1}\right)$ be the Suzuki group over the field with $2^{2 m+1}$ elements, $m>0$. Let $q=2^{2 m+1}$ and $r=2^{m}$. Then

(a) a Sylow 2-subgroup $F$ of $G$ is of order $q^{2}$ and $\left|\left\{F^{x} \mid x \in G\right\}\right|=q^{2}+1$, 
(b) $G$ contains a cyclic subgroup $A$ of order $q-1$ and $\left|\left\{A^{x} \mid x \in G\right\}\right|=$ $q^{2}\left(q^{2}+1\right) / 2$,

(c) $G$ contains a cyclic subgroup $B$ of order $q+2 r+1$ and $\left|\left\{B^{x} \mid x \in G\right\}\right|=$ $q^{2}\left(q^{2}+1\right)(q-1) / 4(q+2 r+1)$,

(d) $G$ contains a cyclic subgroup $C$ of order $q-2 r+1$ and $\left|\left\{C^{x} \mid x \in G\right\}\right|=$ $q^{2}\left(q^{2}+1\right)(q-1) / 4(q-2 r+1)$.

(e) Suppose $x$ is a non-trivial element of $G$. Then,

$$
\operatorname{nil}_{G}(x)= \begin{cases}F^{x} & \text { if } x \in F^{x}, \\ A^{x} & \text { if } x \in A^{x}, \\ B^{x} & \text { if } x \in B^{x}, \\ C^{x} & \text { if } x \in C^{x} .\end{cases}
$$

In particular, $G$ is an $\mathfrak{n n}$-group.

Proof. Parts (a)-(d) follows from [8, Theorem 3.10 of Chapter XI]. By [8, Theorem 3.10 and 3.11 of Chapter XI],

$$
\mathfrak{P}=\left\{A^{x} \backslash\{1\}, B^{x} \backslash\{1\}, C^{x} \backslash\{1\}, F^{x} \backslash\{1\} \mid x \in G\right\}
$$

is a partition for $G$ and $C_{G}(y) \leq M \cup\{1\}$ for all $y \in M$ and $M \in \mathfrak{P}$. Now let $a$ be a nontrivial element of $G$. Since $\mathfrak{P}$ is a partition for $G, a \in M$ for some $M \in \mathfrak{P}$. Now by [2, Lemma 3.7], we have $\operatorname{nil}_{G}(a)=M \cup\{1\}$. These prove (e) and thus it follows that $G$ is an $\mathfrak{n n}$-group.

Proposition 3.8. Let $G=S z\left(2^{2 m+1}\right)$ be the Suzuki group over the field with $2^{2 m+1}$ elements, $m>0$. Then $\lambda\left(\mathfrak{R}_{G}\right)=2$.

Proof. By Proposition 3.7, we have $G$ is an $\mathfrak{n n}$-group. By Remark 3.5, we have $\lambda\left(\mathfrak{R}_{G}\right) \leq 2$. Since $G$ is a simple group, $G$ does not contain a non-trivial proper normal subgroup. Thus Proposition 3.2 completes the proof.

Let $G$ be a non-abelian group. The non-commuting graph of $G$, denoted by $\Gamma(G)$ is the graph with $G \backslash Z(G)$ as vertex set and two vertices are adjacent if they do not commute (see $[1,5])$.

Proposition 3.9. Let $q$ be a prime power number such that $q^{2} \not \equiv 1 \bmod 16$ and $G=P S L(2, q)$. Then $\lambda\left(\mathfrak{R}_{G}\right)=2$.

Proof. By [2, Lemma 3.9], we have $\operatorname{nil}_{G}(a)=C_{G}(a)$ for all non-trivial elements $a \in G$. Thus $\Gamma_{G} \cong \mathfrak{R}_{G}$. Now, [1, Proposition 2.18(1)] completes the proof.

Now we pose the following conjecture. 
Conjecture 3.10. The domination number of the non-nilpotent graph of every finite non-abelian simple group is 2 .

Remark 3.11. The difficulty to prove the conjecture is that, if $G=\langle x, y\rangle$ is a finite simple group, then $\{x, y\}$ need not be a dominating set. For example in the group $P S L(3,2)$, we have, $P S L(3,2)=\langle(3,4)(5,6),(1,2,3,6)(4,7)\rangle$, but $\{(3,4)(5,6),(1,2,3,6)(4,7)\}$ is not a dominating set since $\langle(3,4)(5,6),(1,2)(3,6)\rangle$ and $\langle(1,2,3,6)(4,7),(1,2)(3,6)\rangle$ are nilpotent. Also, by $[7], \lambda\left(\Re_{G}\right)=2$ for any simple group whose order is less than 2000.

Proposition 3.12. Let $G$ be a finite non-nilpotent group. Then $\mathfrak{R}_{G}$ is a complete multi-partite graph if and only if $G$ is an $\mathfrak{n n}$-group. In particular, $\mathfrak{R}_{G} \cong$ $K_{\left|X_{1}\right|, \ldots,\left|X_{n}\right|}$, where $\mathcal{P}=\left\{\operatorname{nil}_{G}(u) \backslash \operatorname{nil}(G) \mid u \in G \backslash \operatorname{nil}(G)\right\}=\left\{X_{1}, \ldots, X_{n}\right\}$.

Proof. Suppose $G$ is an $\mathfrak{n n}$-group. Let $X \in \mathcal{P}$, then $X=n i l_{G}(u) \backslash \operatorname{nil}(G)$ for some $u \in G$. If $x, y \in X$, then $\langle x, y\rangle$ is nilpotent, since $n i l_{G}(u)$ is a nilpotent subgroup. Also, if $x \in X$ and $y \in G \backslash \operatorname{nil}(G)$ such that $\langle x, y\rangle$ is nilpotent, then, we have $\langle u, y\rangle$ is nilpotent, and so, $y \in X$. It follows that $\mathfrak{R}_{G} \cong K_{\left|X_{1}\right|, \ldots,\left|X_{n}\right|}$.

Conversely, suppose $\mathfrak{R}_{G}$ is a complete multipartite graph. Let $X_{1}, X_{2}, \ldots, X_{n}$ be the partite sets. Let $x \in G \backslash \operatorname{nil}(G)$, then $x \in X_{i}$ for some $i$ and $n i l_{G}(x) \backslash$ $\operatorname{nil}(G)=X_{i}$. Now if $y, z \in \operatorname{nil}_{G}(x) \backslash \operatorname{nil}(G)=X_{i}$, then $\langle y, z\rangle$ is nilpotent and $y z \in \operatorname{nil}_{G}(y)=\operatorname{nil}_{G}(x)$. Thus $\left.\operatorname{nil}_{\left(n i l_{G}\right.}(x)\right)=\operatorname{nil}_{G}(x)$ and so $n i l_{G}(x)$ is a nilpotent subgroup of $G$. Thus $G$ is an $\mathfrak{n n}$-group.

Lemma 3.13. Let $G$ be a non-weakly nilpotent group and $x, y \in G$. Then the following assertions hold:

(i) if $\langle x, y\rangle$ nilpotent, then $\left\langle x, y, Z^{*}(G)\right\rangle$ is hypercentral. In particular $\langle x u, y v\rangle$ is nilpotent for all $u, v \in Z^{*}(G)$ and if $G$ is a finite group, then $\left\langle x, y, Z^{*}(G)\right\rangle$ is nilpotent.

(ii) if $\langle x, y\rangle$ is not nilpotent, then for all $u, v \in Z^{*}(G),\langle x u, y v\rangle$ is not nilpotent.

Proof. Let $K=\left\langle x, y, Z^{*}(G)\right\rangle$. Then $Z^{*}(G) \subset Z^{*}(K)$ and

$$
K / Z^{*}(K) \cong\left\langle x Z^{*}(K), y Z^{*}(K)\right\rangle,
$$

a nilpotent group. Therefore $Z^{*}(K)=K$. It follows from [13, Theorem 12.2.3] that $K$ is hypercentral. By [13, Theorem 12.2.4], we have $K$ is locally nilpotent. Thus $\langle x u, y v\rangle$ is nilpotent for all $u, v \in Z^{*}(G)$. If $G$ is a finite group and since $Z^{*}\left(\left\langle x, y, Z^{*}(G)\right\rangle\right)=\operatorname{nil}\left(\left\langle x, y, Z^{*}(G)\right\rangle\right)=\left\langle x, y, Z^{*}(G)\right\rangle$, we have $\left\langle x, y, Z^{*}(G)\right\rangle$ is nilpotent. Part (ii) follows directly from part (i). 
Proposition 3.14. Let $G$ be a non-weakly nilpotent $\mathfrak{n n}$-group and let $S$ be a cut set of $\mathfrak{R}_{G}$. Then $S$ is a union of cosets of $Z^{*}(G)$. In particular if $G$ is finite, then $\kappa\left(\Re_{G}\right)=t|\operatorname{nil}(G)|$, where $t>1$ is an integer.

Proof. Let $a \in S$. Then there exist two distinct components $G_{1}$ and $G_{2}$ of $\mathfrak{R}_{G} \backslash S$ and $x \in G_{1}$ and $y \in G_{2}$ such that $a$ is adjacent to both $x$ and $y$. By Lemma 3.13(ii), $x$ and $y$ are also adjacent to $a z$ for any $z \in Z^{*}(G)$, and so $a Z^{*}(G) \subset S$. Thus $S$ is a union of cosets of $Z^{*}(G)$. Thus the first part follows.

Suppose that $|S|=\kappa\left(\Re_{G}\right)$. It follows from the first part that $\kappa\left(\Re_{G}\right)=t\left|Z^{*}(G)\right|$ for some integer $t \geq 1$. If $t=1$, then $S=\operatorname{bnil}(G)$ for some element $b \in G \backslash \operatorname{nil}(G)$. Then there exist two distinct components $G_{1}$ and $G_{2}$ of $\mathfrak{R}_{G} \backslash S$ and $r \in G_{1}$ and $s \in G_{2}$ such that $b$ is adjacent to both $r$ and $s$. Here, $\langle r, s\rangle$ is nilpotent. Thus $\langle r, r b\rangle$ is not nilpotent. Since $G$ is an $\mathfrak{n n}$-group, we have $\langle s, r b\rangle$ is not nilpotent, which is a contradiction. This completes the proof.

Proposition 3.15. The non-nilpotent graph of every non-nilpotent finite $\mathfrak{n n}$-group is Hamiltonian.

Proof. We first note that the degree of any vertex $x$ in the non-nilpotent graph $\mathfrak{R}_{G}$ of a non-nilpotent group $G$ is equal to $\left|G \backslash n i l_{G}(x)\right|$. Since $x \in G \backslash \operatorname{nil}(G)$ and $G$ is an $\mathfrak{n n}$-group, we have $|G| \geq 2\left|n i l_{G}(x)\right|$. Thus, it follows that $\operatorname{deg}(x)>$ $(|G|-|\operatorname{nil}(G)|) / 2$. Therefore by Dirac's theorem [4, p. 54], $\mathfrak{R}_{G}$ is Hamiltonian.

Remark 3.16. The group $S_{n}, n \geq 4$ is not an $\mathfrak{n n}$-group. To see this, first we note that $S_{4}$ is not an $\mathfrak{n n}$-group, since $\mid$ nil $_{G}((1,2)(3,4))|=16>| G \mid / 2=12$. For $n \geq 5$, $S_{n}$ has a subgroup isomorphic to $S_{4}$. Since the class of $\mathfrak{n n}$-groups are clearly closed under taking subgroups, we have $S_{n}$ is not an $\mathfrak{n n}$-group.

The non-nilpotent graph of $S_{4}$ is Hamiltonian. To see this, we need only to note that the following path is a Hamiltonian cycle of $\mathfrak{R}_{S_{4}}$ :

$(1,2,3)-(1,2)(3,4)-(2,3,4)-(1,3)(2,4)-(2,4,3)-(1,4)(2,3)-(1,4,3)-$ $(1,2,4)-(1,3,4)-(1,3,2)-(1,4,2)-(1,4,2,3)-(1,4,3,2)-(1,3,4,2)-(1,3,2,4)-$ $(1,2,4,3)-(1,2,3,4)-(1,4)-(1,2)-(2,4)-(2,3)-(1,3)-(3,4)-(1,2,3)$

Thus showing that if $\mathfrak{R}_{G}$ is Hamiltonian, then $G$ need not be an $\mathfrak{n n}$-group.

Paul Erdös, posed the following problem in 1975 [9]: Let $G$ be a group whose non-commuting graph has no infinite clique. Is it true that the clique number of $\Gamma(G)$ is finite? Neumann [9] answered positively Erdös question. The dual question of Erdös on non-nilpotent graphs may be posed as the following.

Question 3.17. Let $G$ be a group whose non-nilpotent graph has no infinite independent sets. Is it true that the independence number of $G, \alpha(G)$ is finite? 
The answer is no in general. Consider the disjoint union of cyclic groups of odd order $\mathfrak{C}:=\{\mathbb{Z} / p \mathbb{Z} \mid p$ an odd prime $\}$. By a theorem of A. Yu. Ol'shanskii (see [10, Theorem 35.1]), there exists an odd integer $n$ for which this collection of groups can be embedded into a countable 2-generated simple group $G$ such that every proper subgroup of $G$ either is cyclic of order dividing $n$ or is conjugate to a subgroup of a group from $\mathfrak{C}$.

Clearly $G$ is non-nilpotent, but every proper subgroup of $G$ is cyclic of finite odd prime order. Since each group from $\mathfrak{C}$ is a subgroup of $G$, one sees that there is no bound on the orders of the cyclic subgroups.

We show in the next theorem that the answer to the above question is yes in some cases.

Theorem 3.18. Let $G$ be a non-weakly nilpotent group whose non-nilpotent graph has no infinite independent sets. If nil $(G)$ is a subgroup and $G$ is an Engel, locally finite, locally solvable or a linear group or a 2-group, then $G$ is a finite group. In particular $\alpha(G)$ is finite.

Proof. If $x \in G \backslash \operatorname{nil}(G)$, then $\operatorname{xnil}(G)$ is an independent set of $G$. Thus $\operatorname{nil}(G)$ is finite. Therefore, the hypothesis is equivalent to the following: Every nilpotent subgroup of $G$ is finite and thus every abelian subgroup of $G$ is finite. In particular, every cyclic subgroup of $G$ is finite, so $G$ is periodic. We know that an infinite locally finite group or an infinite 2-group contains an infinite abelian subgroup (see [13, Theorem 14.3.7]), and also every periodic locally solvable or linear group is locally finite. Thus in these case $G$ is finite. Now if $G$ is Engel group, since every abelian subgroup of $G$ is finite, a result of Plotkin [12, Corollary, p. 55], implies that $G$ is a finite nilpotent group. This completes the proof.

Proposition 3.19. Let $G$ be an $\mathfrak{n n}$-group. Then for every maximal independent set $S, S \cup \operatorname{nil}(G)$ is a maximal weakly nilpotent subgroup of $G$.

Proof. We have $S \cup \operatorname{nil}(G) \subset \operatorname{nil}_{G}(x)$ for all $x \in S$. Since $S$ is maximal and $G$ is an $\mathfrak{n n}$-group, we have

$$
\bigcap_{x \in S} \operatorname{nil}_{G}(x)=S \cup \operatorname{nil}(G) .
$$

is a maximal weakly nilpotent subgroup of $G$.

Remark 3.20. Consider the group

$G=\left\langle x, y, z \mid x^{2}=y^{2}=z^{3}=(x y)^{4}=(x z)^{2}=1,(y z)^{2}=(z y)^{2},(x y)^{2} z(x y)^{2}=z^{-1}\right\rangle$. 
Then $G$ is not an $\mathfrak{n n}$-group. Also $I=\{x, y, y x z y\}$ is an independent set of $\mathfrak{R}_{G}$ and $\langle I\rangle \cong G$. This shows that a subgroup generated by an independent set may not be a nilpotent subgroup. Also there exists a maximal independent set $S$, such that $X \subseteq S$. Since the edge set of $\mathfrak{R}_{G}$ is non-empty, we have $S \neq G \backslash \operatorname{nil}(G)$, showing that $S \cup \operatorname{nil}(G)$ is not a subgroup of $G$. So the last proposition cannot, in general, be improved.

\section{Groups with the same non-nilpotent graphs}

In this section we consider the non-weakly nilpotent groups with isomorphic non-nilpotent graphs. Note that if $G$ and $H$ are two groups, then $\mathfrak{R}_{G} \cong \mathfrak{R}_{H}$ if and only if there exists a bijective map $\phi: V\left(\mathfrak{R}_{G}\right) \longrightarrow V\left(\mathfrak{R}_{H}\right)$ such that for every two distinct elements $x, y \in V\left(\mathfrak{R}_{G}\right)$, we have $\langle x, y\rangle$ is not nilpotent if and only if $\langle\phi(x), \phi(y)\rangle$ is not nilpotent.

We begin the section with the following theorem.

Theorem 4.1. Let $G$ and $H$ be two groups such that $\mathfrak{R}_{G} \cong \mathfrak{R}_{H}$. If $G$ is finite non-nilpotent group, then $H$ is also a finite non-nilpotent group. Moreover $\mid$ nil $(H) \mid$ divides

$$
\operatorname{gcd}\left(|G|-|\operatorname{nil}(G)|,|G|-\left|\operatorname{nil}_{G}(x)\right|,\left|\operatorname{nil} l_{G}(x)\right|-|\operatorname{nil}(G)|: x \in G \backslash \operatorname{nil}(G)\right) .
$$

Proof. Since $\mathfrak{R}_{G} \cong \mathfrak{R}_{H}$, we have $|H \backslash \operatorname{nil}(H)|=|G \backslash \operatorname{nil}(G)|$ and $|H \backslash \operatorname{nil}(H)|$ is finite. If $h \in H \backslash \operatorname{nil}(H)$, then $\left\{h^{x} \mid x \in H\right\} \subset H \backslash \operatorname{nil}(H)$, since $\operatorname{nil}(H)$ is closed under conjugation. Thus every element in $H \backslash \operatorname{nil}(H)$ has finitely many conjugates in $H$. It follows that $K=C_{H}(H \backslash \operatorname{nil}(H))$ has finite index in $H$. Now, $\mathfrak{R}_{H}$ has no isolated vertex. Thus there exist two adjacent vertices $u$ and $v$ in $\mathfrak{R}_{H}$. Now if $s \in K$, then $s \in C_{H}(u, v)$. It follows that $\langle s u, v\rangle$ is not nilpotent. Therefore $K u \subset H \backslash \operatorname{nil}(H)$ and so $K$ is finite. Hence $H$ is a finite non-nilpotent group.

Now since $H$ is finite, it follows that $\operatorname{nil}(H)$ is a subgroup of $H$ and so $|\operatorname{nil}(H)|$ divides $|H|-|\operatorname{nil}(H)|$. Since $|H|-|\operatorname{nil}(H)|=|G|-|\operatorname{nil}(G)|$, we have $|\operatorname{nil}(H)|$ divides $|G|-|\operatorname{nil}(G)|$. Let $x \in H \backslash \operatorname{nil}(H)$ and $y \in \operatorname{nil}_{H}(x)$. Then, by Lemma 3.13, $\langle x, y z\rangle$ is nilpotent for all $z \in \operatorname{nil}(H)$. Thus $\operatorname{nil}_{H}(x)=\operatorname{nil}(H) \cup y_{1} \operatorname{nil}(H) \cup \cdots \cup y_{n} n i l(H)$, for some $y_{i} \in H$. Therefore $|\operatorname{nil}(H)|$ divides $\left|n i l_{H}(x)\right|$ and so $|\operatorname{nil}(H)|$ divides $|H|-\left|n i l_{H}(x)\right|$. Now since $\mathfrak{R}_{G} \cong \mathfrak{R}_{H},\left\{\operatorname{deg}(v) \mid v \in V\left(\mathfrak{R}_{G}\right)\right\}=\{\operatorname{deg}(v) \mid v \in$ $\left.V\left(\mathfrak{R}_{H}\right)\right\}$. But $\operatorname{deg}(v)=|G|-\left|n i l_{G}(v)\right|$ for any $v \in V\left(\mathfrak{R}_{G}\right)$ and $\operatorname{deg}(v)=|H|-$ $\left|n i l_{H}(v)\right|$ for any $v \in V\left(\Re_{H}\right)$. It follows that $|\operatorname{nil}(H)|$ divides $|G|-\left|n i l_{G}(x)\right|$ for any $x \in G \backslash \operatorname{nil}(G)$. Since $|\operatorname{nil}(H)|$ divides $|G|-|\operatorname{nil}(G)|$ and $|G|-\left|\operatorname{nil}_{G}(x)\right|$, it 
divides $|G|-|\operatorname{nil}(G)|-\left(|G|-\left|\operatorname{nil}_{G}(x)\right|\right)=\left|\operatorname{nil}_{G}(x)\right|-|\operatorname{nil}(G)|$. This completes the proof.

Proposition 4.2. Let $G$ be a non-weakly nilpotent group such that $\mathfrak{R}_{G}$ is finite. Then $G$ is a finite group.

Proof. It follows directly from the first paragraph of the proof of the above theorem.

Proposition 4.3. Let $G$ a finite non-nilpotent group and $H$ be a group. Suppose $\omega\left(\mathfrak{R}_{G}\right)=4$ and $\mathfrak{R}_{G} \cong \mathfrak{R}_{H}$, then $|G|=|H|$.

Proof. Since $\mathfrak{R}_{G} \cong \mathfrak{R}_{H}$, by Theorem 4.1, we have $H$ is a finite group. Since $\omega\left(\mathfrak{R}_{G}\right)=4$, we have $\omega\left(\mathfrak{R}_{H}\right)=4$ and by [2, Theorem 4.7], we have $\frac{G}{Z^{*}(G)} \cong$ $S_{3} \cong \frac{H}{Z^{*}(H)}$. Now, $\frac{G}{Z^{*}(G)}=\left\langle\bar{x}, \bar{y} \mid \bar{x}^{2}=\bar{y}^{3}=1, \bar{x} \bar{y} \bar{x}=\bar{y}^{-1}\right\rangle$, where $\bar{x}=$ $x Z^{*}(G), \bar{y}=y Z^{*}(G)$, for some $x, y \in G$. Then $G=x Z^{*}(G) \cup y Z^{*}(G) \cup y^{2} Z^{*}(G) \cup$

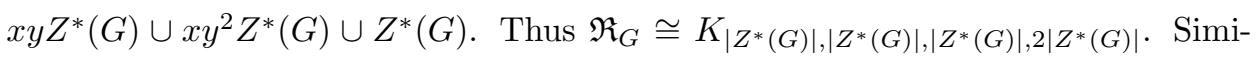

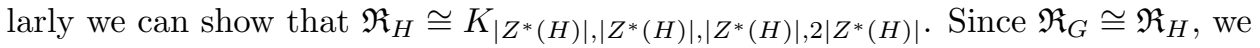
have $\left|Z^{*}(G)\right|=\left|Z^{*}(H)\right|$. Also $|G|-\left|Z^{*}(G)\right|=|H|-\left|Z^{*}(H)\right|$ and so $|G|=|H|$.

Proposition 4.4. Let $n>2$ be an integer and not a power of 2 and $G=D_{2 n}$. If $\mathfrak{R}_{G} \cong \mathfrak{R}_{H}$, for some group $H$, then $|G|=|H|$ and $H$ contains a nilpotent subgroup of index 2.

Proof. It is enough to show that $\left|Z^{*}(G)\right|=\left|Z^{*}(H)\right|$. Let $n=2^{t} m$, where $m$ is odd. We have, $|\operatorname{nil}(G)|=2^{t}$ and there exist $x, y \in G$ such that $\left|n i l_{G}(x)\right|=2^{t} m$ and $\left|n i l_{G}(y)\right|=2^{t+1}$. Thus $2^{t+1}-2^{t}=\left|n i l_{H}(u)\right|-|\operatorname{nil}(H)|$ for some $u \in H \backslash \operatorname{nil}(H)$. Thus we find that $|\operatorname{nil}(H)|$ divides $2^{t}$ and so $|\operatorname{nil}(H)|=2^{l}$ for some $l \leq t$. Now, $|H|=2^{t+1} m-2^{t}+2^{l}$ and $2^{t+1} m-2^{t} m=2^{t+1} m-2^{t}+2^{l}-\left|n i l_{H}(v)\right|$ for some $v \in H \backslash \operatorname{nil}(H)$. Therefore $\left|n i l_{H}(v)\right|=m 2^{t}-2^{t}+2^{l}$. Since $G$ is an $\mathfrak{n n}$-group, by Proposition 3.12, we have $H$ is also an $\mathfrak{n n}$-group and so $m 2^{t}-2^{t}+2^{l}$ divides $2^{t+1} m-2^{t}+2^{l}$. It follows that $2^{l}\left(2^{t-l}(m-1)+1\right)$ divides $2^{t+1} m-2^{t}+2^{l}-2\left(m 2^{t}-\right.$ $\left.2^{t}+2^{l}\right)=2^{t}-2^{l}$. If $l \neq t$, then $2^{t} m-2^{t}+2^{l} \nmid 2^{t}-2^{l}$, which is a contradiction. Thus $l=t$ and so $|G|=|H|$.

Now since $G$ has a cyclic subgroup $A$ of order $2 m$ (which is a maximal abelian subgroup), $\mathfrak{R}_{G} \cong \mathfrak{R}_{H}$ and $|G|=|H|$, Proposition 3.19 implies that $B=\varphi(A \backslash$ $\operatorname{nil}(G)) \cup \operatorname{nil}(H)$ is a nilpotent subgroup of order $2 m$ in $H$, where $\varphi$ is a graph isomorphism from $\mathfrak{R}_{G}$ to $\mathfrak{R}_{H}$. Clearly $B$ is of index 2 in $H$, as required.

Lemma 4.5. Let $G$ be a centerless AC-group. Then the non-commuting graph and the non-nilpotent graph of $G$ are isomorphic. 
Proof. Since $Z(G)=\operatorname{nil}(G)=1$, we have $G \backslash Z(G)=V(\Gamma(G))=V\left(\mathfrak{R}_{G}\right)=$ $G \backslash \operatorname{nil}(G)$. By [2, Lemma 3.6], we have, for $x, y \in G,\langle x, y\rangle$ is not-nilpotent if and only if $x$ and $y$ do not commute. Thus the result follows.

Proposition 4.6. Let $q$ be a prime power number such that $q^{2} \not \equiv 1 \bmod 16$ and $G=P S L(2, q)$. If $\mathfrak{R}_{G} \cong \mathfrak{R}_{H}$, then $|G|=|H|$. Moreover, if $q=2^{k}, k \geq 1$ and $H$ is an AC-group, then $G \cong H$.

Proof. Note that $|\operatorname{nil}(G)|=1$, so it is enough to show that $|\operatorname{nil}(H)|=1$. By $[2$, Lemma 3.9], we have $n i l_{G}(a)=C_{G}(a)$ for all non-trivial elements $a \in G$. By [1, Lemma 3.22], there exist two non-trivial element $x, y \in G$ such that $\left|n i l_{G}(x)\right|-$ $\left|n i l_{G}(y)\right|=1$. By Theorem $4.1, H$ is a finite group and $|n i l(H)|$ divides 1 . Thus $|G|=|H|$.

If $k=1$, then $G \cong S_{3}$. Since $|G|=|H|$, we have $G \cong H$. So assume that $k>1$, then by [1, Proposition 3.21], $G$ is a centerless AC-group and so $H$ is also a centerless AC-group. Since $\mathfrak{R}_{G} \cong \mathfrak{R}_{H}$ and both the group are centerless AC-group, we have, by Lemma 4.5, $\Gamma(G) \cong \Gamma(H)$. Thus the result follows from [16]. This completes the proof.

Proposition 4.7. Let $G$ be a non-nilpotent group of order $p q$, where $p$ and $q$ are primes with $q>p$. If $\mathfrak{R}_{G} \cong \mathfrak{R}_{H}$ for some group $H$, then $|G|=|H|$. In particular $G \cong H$.

Proof. The group $G$ is an $\mathfrak{n n}$-group with $\left|Z^{*}(G)\right|=1$ and there exists $x \in G$, such that $\mid$ nil $_{G}(x) \mid=q$. Thus $\left|Z^{*}(H)\right|$ divides $q-1$. Suppose $\left|Z^{*}(H)\right|=l$. Then $|G|-\left|Z^{*}(G)\right|=|H|-\left|Z^{*}(H)\right|$ and thus $|H|=p q-1+l$. Now, since $\mathfrak{R}_{G} \cong \mathfrak{R}_{H}$, there exists $u \in H$, such that $p q-q=p q-1+l-\left|n i l_{H}(u)\right|$. Since $n i l_{H}(u)$ is a subgroup of $H$, we have $\left|n i l_{H}(u)\right|=l+q-1$ divides $p q-1+l$ and thus it divides $p q-q$. Since $q$ is a prime, we have, either $q \mid l+q-1$ or $q \nmid l+q-1$. Since $l+q-1>p-1$, we have $q \mid l+q-1$ and thus $q \mid l-1$. Since $l \mid q-1$, we have $l=1$. Thus $|G|=|H|$. Since there is only one non-nilpotent group of order $p q$, up to isomorphism, we have $G \cong H$.

Proposition 4.8. Let $G$ be a centerless non-nilpotent group of order pqr, where $p, q$ and $r$ are primes, with $p<q<r$. Suppose there exists $x \in G \backslash\{1\}$, such that $\left|n i l_{G}(x)\right|=$ qr. If $\mathfrak{R}_{G} \cong \mathfrak{R}_{H}$ for some group $H$, then $|G|=|H|$.

Proof. Let $x \in G \backslash\{1\}$. Then $\left|C_{G}(x)\right|=\bar{p}$ or $\bar{p} \bar{q}$, where $\bar{p}, \bar{q} \in\{p, q, r\}$. If $\left|C_{G}(x)\right|=\bar{p}$, then $C_{G}(x)$ is abelian. So suppose $\left|C_{G}(x)\right|=\bar{p} \bar{q}$. Then $\left|Z\left(C_{G}(x)\right)\right|=\bar{p}$ or $\bar{q}$ or $\bar{p} \bar{q}$. Thus $C_{G}(x) / Z\left(C_{G}(x)\right)$ is cyclic and so $C_{G}(x)$ is abelian. Thus the group 
$G$ is a centerless $A C$-group and thus by [2, Lemma 3.6], $G$ is an $\mathfrak{n n}$-group. It follows from Proposition 3.12 that $H$ is an $\mathfrak{n n}$-group. Given that there exists $x \in G$, such that $\left|n i l_{G}(x)\right|=q r$. Now $\left|Z^{*}(H)\right|$ divides $p q r-1$. Suppose $\left|Z^{*}(H)\right|=l$. Then $|G|-\left|Z^{*}(G)\right|=|H|-\left|Z^{*}(H)\right|$ and thus $|H|=p q r-1+l$. Now, since $\mathfrak{R}_{G} \cong \mathfrak{R}_{H}$, there exists $u \in H$, such that $p q r-q r=p q r-1+l-\left|n i l_{H}(u)\right|$. Since $n i l_{H}(u)$ is a subgroup of $H$, we have $\left|n i l_{H}(u)\right|=l+q r-1$ divides $p q r-q r$. Since $q$ and $r$ are primes and $q r+l-1>(p-1) q,(p-1) r$, we have, $q \mid l+q r-1$ and $r \mid l+q r-1$. Thus $q r \mid l-1$. But $l \mid q r-1$. Thus $l=1$ and $|G|=|H|$.

Remark 4.9. The non-nilpotent graph of the following groups,

$$
\begin{aligned}
& G=\left\langle x, y, x \mid x^{3}=y^{7}=z^{13}=e, z y=y z, x^{-1} y x=y^{2}, x^{-1} z x=y^{3}\right\rangle, \\
& H=\left\langle x, y, x \mid x^{3}=y^{7}=z^{13}=e, z y=y z, x^{-1} y x=y^{4}, x^{-1} z x=y^{3}\right\rangle,
\end{aligned}
$$

are isomorphic to a complete 92-partite graph, with 91 partite set of size 2 and one partite set of size 90. Also $\left\{\left|n i l_{G}(x)\right| \mid x \in G \backslash \operatorname{nil}(G)\right\}=\left\{\left|\operatorname{nil}_{H}(x)\right| \mid x \in\right.$ $H \backslash \operatorname{nil}(H)\}=\{3,7 \cdot 13\}$. But $G \supsetneqq H$. So the last proposition cannot be improved.

Remark 4.10. Suppose that $G$ and $H$ are two non-abelian groups. If $\mathfrak{R}_{G} \cong \mathfrak{R}_{H}$, then $\mathfrak{R}_{G \times A} \cong \mathfrak{R}_{H \times B}$, for any two weakly nilpotent groups $A$ and $B$ with the same order. To see this, suppose $\varphi: \mathfrak{R}_{G} \rightarrow \mathfrak{R}_{H}$ be a graph isomorphism and $\psi: A \rightarrow B$ be a bijective map. Then it is easy to see that $\phi:(g, a) \mapsto(\varphi(g), \psi(a))$ is a graph isomorphism between $\mathfrak{R}_{G \times A}$ and $\mathfrak{R}_{H \times B}$.

We call a non-weakly nilpotent group $G$ an $F n$-group if for every two elements $x, y \in G \backslash n i l(G)$, such that $\operatorname{nil}_{G}(x) \neq n i l_{G}(y)$, we have $n i l_{G}(x) \not \subset n i l_{G}(y)$ and $\operatorname{nil}_{G}(y) \not \subset n i l_{G}(x)$.

Proposition 4.11. Let $G$ be a non-weakly nilpotent Fn-group. If $H$ is a group such that $\mathfrak{R}_{G} \cong \mathfrak{R}_{H}$, then $H$ is also a non-weakly nilpotent Fn-group.

Proof. Let $x, y \in H \backslash n i l(H)$ and $n i l_{H}(x) \subseteq n i l_{H}(y)$, then $\psi\left(n i l_{H}(x) \backslash n i l(H)\right) \subseteq$ $\psi\left(n i l_{H}(y) \backslash \operatorname{nil}(H)\right)$, where $\psi: V\left(\mathfrak{R}_{H}\right) \rightarrow V\left(\mathfrak{R}_{G}\right)$ is a graph isomorphism. Thus, since $G$ is an $F n$-group, we have $\operatorname{nil}_{G}(\psi(x)) \backslash \operatorname{nil}(G)=n i l_{G}(\psi(y)) \backslash \operatorname{nil}(G)$. It follows that $n i l_{H}(x) \backslash n i l(H)=\operatorname{nil}_{H}(y) \backslash n i l(H)$ and so $n i l_{H}(x)=n i l_{H}(y)$. Thus $H$ is an $F n$-group.

\section{Genus of non-nilpotent graph}

In this section, we study the genus of the non-nilpotent graph $\mathfrak{R}_{G}$ for a nonweakly nilpotent group $G$. 
Lemma 5.1. Let $G$ be a finite non-nilpotent group of genus $\gamma$. Then $\left|Z^{*}(G)\right| \leq$ $\sqrt{\gamma}+1$

Proof. Assume $Z=Z^{*}(G)$. Since $G$ is a finite non-weakly nilpotent group, by $\left[2\right.$, Theorem 4.2], we have $\omega\left(\mathfrak{R}_{G}\right) \geq 4$. So there exist $u, v, w, x \in G \backslash Z$, such that they are adjacent to each other. Then, by Lemma 3.13, $\mathfrak{R}_{G}[u Z \cup v Z \cup w Z \cup x Z]$

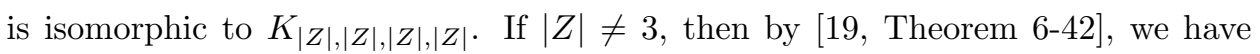
$\gamma\left(K_{|Z|,|Z|,|Z|,|Z|}\right)=(|Z|-1)^{2} \leq \gamma$. Thus $|Z| \leq \sqrt{\gamma}+1$. Also if $\left|Z^{*}(G)\right|=3$, then $K_{6,6}$ is a subgraph of $K_{3,3,3,3}$. Thus $\gamma\left(K_{6,6}\right)=4 \leq \gamma$ and so $\left|Z^{*}(G)\right| \leq \sqrt{\gamma}+1$. This completes the proof.

Remark 5.2. It is easy to see that $\mathfrak{R}_{D_{12}} \cong \mathfrak{R}_{Q_{12}} \cong K_{4,2,2,2}=H$. Since $K_{4,6}$ is a subgraph of $H$ and also $H$ is a subgraph of $K_{10}$, we have $2 \leq \gamma\left(\Re_{D_{12}}\right)=$ $\gamma\left(\Re_{Q_{12}}\right) \leq 4$. Now, $A_{4}$ is a centerless AC-group and thus by Lemma 4.5, we have $\Gamma\left(A_{4}\right) \cong \mathfrak{R}_{A_{4}}$. Thus, since $A_{4}$ has five distinct centralizers of size $4,3,3,3,3$, we have $\mathfrak{R}_{A_{4}} \cong K_{3,2,2,2,2}$. It is easy to see that $\mathfrak{R}_{A_{4}}$ has 48 edges and 11 vertices. Thus by [19, Corollary 6-14], we have $\gamma\left(\mathfrak{R}_{A_{4}}\right) \geq \frac{48}{6}-\frac{11}{2}+1=3.5$. Also $\mathfrak{R}_{A_{4}}$ is a subgraph of $K_{11}$ and so $4 \leq \gamma\left(\Re_{A_{4}}\right) \leq 5$.

Proposition 5.3. Let $G$ be a finite non-nilpotent group. Then $\mathfrak{R}_{G}$ is not toroidal.

Proof. Suppose $\mathfrak{R}_{G}$ is toroidal. By Lemma 5.1, we have $\left|Z^{*}(G)\right| \leq 2$. We will show that $Z^{*}(G)=1$. Suppose for a contradiction that $\left|Z^{*}(G)\right|=2$. Let $H$ be a clique of $\mathfrak{R}_{G}$ and $z \in Z^{*}(G) \backslash\{e\}$. Now, by Lemma 3.13 , the induced subgraph $\mathfrak{R}_{G}[z V(H)]$ is a clique of $\mathfrak{R}_{G}$, where $V(H)$ is the vertex set of $H$. Then $H$ and $\mathfrak{R}_{G}[z V(H)]$ are two distinct cliques of $\mathfrak{R}_{G}$. Now the subgraph $K=H \cup \mathfrak{R}_{G}[z V(H)]$ has two components. Since $K$ is a subgraph of $\mathfrak{R}_{G}$, we have $1=\gamma\left(\mathfrak{R}_{G}\right) \geq \gamma(K)=\gamma(H)+$ $\gamma\left(\mathfrak{R}_{G}[z V(H)]\right)=2 \gamma\left(K_{|V(H)|}\right)$. It follows that $|V(H)| \leq 4$ and so $\omega\left(\Re_{G}\right) \leq 4$. By $[2$, Theorem 4.2] we have $\omega\left(\Re_{G}\right)=4$ and by [2, Theorem 4.7] we have $G / Z^{*}(G) \cong S_{3}$. Thus $|G|=12$ and $G \cong Q_{12}$ or $D_{12}$. Now by Remark 5.2 , we get a contradiction. Thus $Z^{*}(G)=1$.

Next we will show that for all $g \in G$, order of $g$ is either 1 or 2 or 3 . Suppose for a contradiction that there exists $g \in G$, such that $\circ(g) \geq 5$. Then there exist $x, y \in G$, such that $\left\langle g^{2}, x\right\rangle$ and $\langle g, y\rangle$ are not nilpotent. If $\circ(g)=6$, then let $H=\left\{g, g^{2}, g^{4}, g^{5}\right\}$ and $L=x H \cup\{x\}$; otherwise $H=\left\{g, g^{i_{1}}, g^{i_{2}}, g^{i_{3}}\right\}$, where $i_{j}>1$ and $\operatorname{gcd}\left(\circ(g), i_{j}\right)=1$ and $L=y H \cup\{y\}$. Then the induced subgraph $\mathfrak{R}_{G}[H \cup L]$ is isomorphic to $K_{4,5}$, which is a contradiction. Suppose there exist $g, h \in G$, such that $\circ(g)=\circ(h)=4$ and $g \neq h, h^{-1}$. Then there exist $x, y \in G$, such that $\left\langle g^{2}, x\right\rangle$ and $\left\langle h^{2}, y\right\rangle$ are not nilpotent. Let $H=\left\{g, g^{2}, g^{3}\right\}$ and $L=\left\{h, h^{2}, h^{3}\right\}$. Then the 
induced subgraph $\mathfrak{R}_{G}[H \cup x H]$ and $\mathfrak{R}_{G}[L \cup y L]$ are disjoint and are isomorphic to $K_{3,3}$, which is a contradiction. Thus $G$ has no element of order 4 , since $Z(G)=1$. Thus for all $g \in G$, order of $g$ is either 1 or 2 or 3 .

Let $H$ and $K$ be the union of all the 2-elements and 3-elements of $G$, respectively. Let $x \in H$ and $y \in K$. Then,

$$
\langle x, y\rangle \cong \begin{cases}S_{3} & \text { if } \circ(x y)=2, \\ Q_{12} & \text { if } \circ(x y)=3 .\end{cases}
$$

Thus $\langle x, y\rangle$ is not nilpotent and so $K_{|H|,|K|}$ is a subgraph of $\mathfrak{R}_{G}[H \cup K]$.

Let $G_{2}$ and $G_{3}$ be a Sylow 2-subgroup and a Sylow 3-subgroup of $G$, respectively. Note that not both $G_{2}$ and $G_{3}$ are normal, otherwise the group is abelian. Suppose $G_{2}$ is normal in $G$. Then $G_{3}$ is not normal in $G$. Suppose $\left|G_{2}\right|=2$. Then $y x y^{-1}=x$ for $x \in G_{2} \backslash\{1\}$ and so $G_{2} \leq Z(G)=\{1\}$, a contradiction. So $\left|G_{2}\right| \geq 4$ and $|H| \geq 3$. So the number of Sylow 3-subgroup of $G$ is at least 4 . Thus $|K| \geq 8$ and $K_{3,8}$ is a subgraph of $\mathfrak{R}_{G}$. But $\gamma\left(K_{3,8}\right)>1$, a contradiction. Thus $G_{2}$ is not normal in $G$.

Suppose for a contradiction that $\left|G_{2}\right| \geq 8$. Let $P_{1}, P_{2}$ and $P_{3}$ be three distinct Sylow 2-subgroups of $G$. Let $U=\left(P_{1} \cap P_{2}\right) \cup\left(P_{2} \cap P_{3}\right) \cup\left(P_{1} \cap P_{3}\right)$ and $Q_{1}=P_{1} \backslash U$, $Q_{2}=P_{2} \backslash U$ and $Q_{3}=P_{3} \backslash U$. Now for any $x, y \in\left(P_{1} \cup P_{2} \cup P_{3}\right) \backslash\{1\}$, we have $\circ(x y)=2$ or 3 . If $\circ(x y)=2$, then $x$ and $y$ commute and if $\circ(x y)=3$, then $\langle x, y\rangle \cong$ $S_{3}$. Thus $n i l_{P_{i}}(x)=C_{P_{i}}(x)$. Now, for $x \in Q_{i}$ and $i \neq j$, if $C_{P_{j}}(x)=P_{j}$, then $\left\langle x, P_{j}\right\rangle$ is a 2-group and $\left|P_{j}\right|<\left|\left\langle x, P_{j}\right\rangle\right|$, which is absurd. Therefore $C_{P_{j}}(x) \neq P_{j}$. Thus $\operatorname{deg}(x) \geq\left|P_{r} \backslash n i l_{P_{r}}(x)\right|+\left|P_{s} \backslash n i l_{P_{s}}(x)\right| \geq 4+4=8$, where $r, s \in\{1,2,3\} \backslash\{i\}$ and $r \neq s$. Now, consider the subgraph $S=\mathfrak{R}_{G}\left[\left(P_{1} \cup P_{2} \cup P_{3}\right) \backslash U\right]$. Then each vertex of $S$ has degree at least 8 and $S$ has genus at most 1 . Now by [20, Proposition 2.1 , we get a contradiction.

Since $G_{2}$ is not normal in $G$, the number of Sylow 2 -subgrops is at least 3 . It follows that $|H| \geq 3$ and since $\mathfrak{R}_{G}$ is toroidal, we have $\gamma\left(K_{|3|,|K|}\right) \leq 1$. Therefore $|K|=2$ and $\left|G_{3}\right|=3$. Therefore $\left|G_{2}\right| \leq 12$. The only centerless group of order less than or equal to 12 are $S_{3}, D_{10}$ and $A_{4}$ and by Remark 5.2 and Proposition 5.6, $A_{4}$ and $D_{10}$ are not toroidal. On the other hand, by [2, Theorem 6.1], the group $S_{3}$ is planar. This completes the proof.

We need the following result in the sequel.

Proposition 5.4. Let $G$ be a group whose non-commuting graph of $G$ is of genus $\gamma$, where $\gamma$ is a non-negative integer. Then $G$ is a finite group and $|G|$ and $|Z(G)|$ satisfy the inequality, $(|G|-12)(|G|-|Z(G)|) \leq 24 \gamma-24$. Thus the number of groups up to isomorphism whose non-commuting graph is of genus $\gamma$ is finite. 


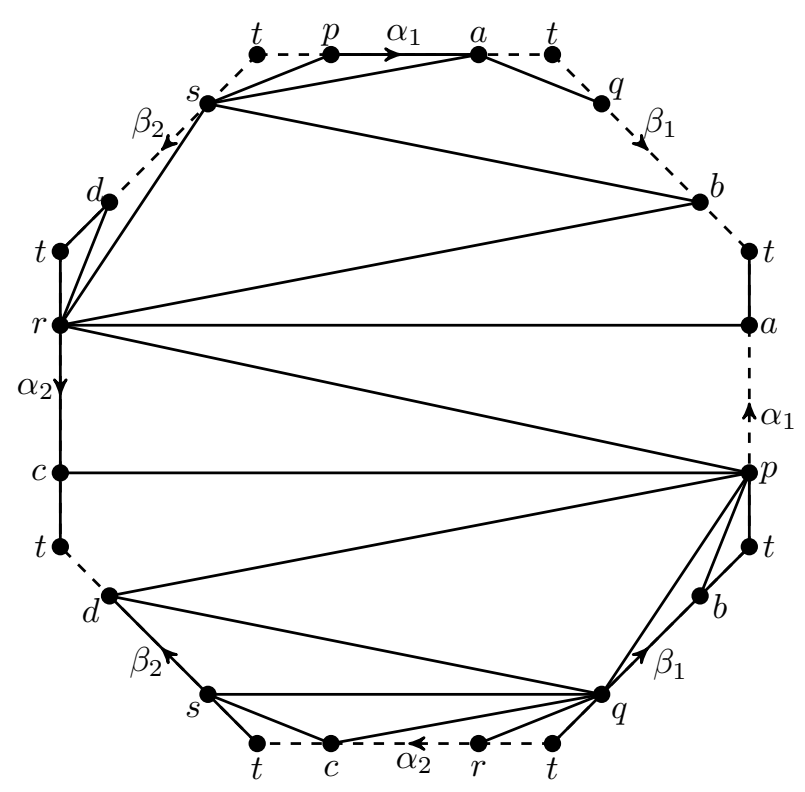

Figure 1. Embedding of the non-commuting graph $\Gamma\left(D_{10}\right)$ on a double torus.

Proof. Let $C$ be a clique of $\Gamma(G)$. Let $H$ be a finite subset of $C$. Thus $H$ is of genus at most $\gamma$. If $\gamma=0$, then $|H| \leq 4$. Therefore by [18, Proposition 6.3.25,], we have $|H|=\omega(H) \leq \chi(H) \leq\left|\frac{7+\sqrt{1+48 \gamma}}{2}\right|=h$. Therefore every clique of $\Gamma(G)$ contain at most $h$ elements and thus $G / Z(G)$ is a finite group, by main result of [11]. Next we show that $Z(G)$ is finite. Let $Z \subset Z(G)$, such that $|Z|<\infty$. Since $G$ is a non-abelian group, there exist $x, y \in G$, such that $x y \neq y x$. Consider the induce subgraph $T$ by the set $Z x \cup Z y$. Thus $T$ is of genus at most $\gamma$. Now for any $z_{1}, z_{2} \in Z$, we have $z_{1} x$ does not commute with $z_{2} y$. Thus $T \cong K_{|Z|,|Z|}$ and $\gamma(T)=\left\lceil\frac{(|Z|-2)(|Z|-2)}{4}\right\rceil \leq \gamma$. Thus $Z(G)$ is finite and therefore $G$ is a finite group.

Let $n=|V(\Gamma(G))|$. Now by [20, Proposition 2.1], we have $\delta(\Gamma(G)) \leq 6+\frac{12 \gamma-12}{n}$. So there exists $x \in G$, such that $\operatorname{deg}(x) \leq 6+\frac{12 \gamma-12}{n}$. We have $\operatorname{deg}(x)=\left|G \backslash C_{G}(x)\right|$. Thus $|G| \leq 6+\frac{12 \gamma-12}{n}+\left|C_{G}(x)\right|$. Note that $n=|G|-|Z(G)|$ and $\left|C_{G}(x)\right| \leq|G| / 2$. Therefore $|G| \leq 6+\frac{12 \gamma-12}{|G|-|Z(G)|}+\frac{|G|}{2}$ and so $(|G|-12)(|G|-|Z(G)|) \leq 24 \gamma-24$.

We need the following result in the sequel.

Proposition 5.5. Let $G$ be a non-abelian group and $\Gamma(G)$ is double-toroidal. Then $G$ is isomorphic to $D_{10}$, Dic $c_{12}$ or $D_{12}$. Moreover, the non-commuting graph of $D_{10}$ is double-toroidal. 
Proof. Suppose that $\Gamma(G)$ is double-toroidal. Putting $\gamma=2$ in the inequality of Proposition 5.4, we get $|G| \leq 14$. Now by [1, Proposition 2.3], we have nonabelian group of order less than or equal to 8 are planar. Thus $|G|=10,12$ or 14. Now groups of order 10,12 or 14 are $D_{10}, D i c_{12}, A_{4}, D_{12}$ and $D_{14}$. Suppose $\Gamma(G) \cong A_{4}$. Then $n=|V(\Gamma(G))|=11$. Now by [20, Proposition 2.1], we have $\delta(\Gamma(G)) \leq 6+\frac{12 \gamma-12}{n} \leq 7$. But for all $v \in V(\Gamma(G))$, we have $\operatorname{deg}(v) \geq 8$, which is a contradiction. Thus $A_{4}$ is not double-toroidal. Suppose $\Gamma(G) \cong D_{14}$. Then $n=$ $|V(\Gamma(G))|=13$. Now by [20, Proposition 2.1], we have $\delta(\Gamma(G)) \leq 6+\frac{12 \gamma-12}{n} \leq 6$. But for all $v \in V(\Gamma(G))$, we have $\operatorname{deg}(v) \geq 7$, which is a contradiction. Thus $D_{14}$ is not double-toroidal.

The non-commuting graph $D_{10}$ is isomorphic to $K_{4,1,1,1,1,1}$. Let the partite sets of $K_{4,1,1,1,1,1}$ be $\{a, b, c, d\},\{p\},\{q\},\{r\}$ and $\{s\}$. Then, the graph $K_{4,1,1,1,1,1}$ can be embedded on a double torus as shown in figure 1 . Thus, $D_{10}$ is double-toroidal. This completes the proof.

Proposition 5.6. Let $G$ be a centerless AC-group which is not weakly nilpotent. Then $\mathfrak{R}_{G}$ is double-toroidal if and only if $G \cong D_{10}$.

Proof. It follows from Lemma 4.5 and Proposition 5.5.

\section{Clique number and chromatic number of some group}

Proposition 6.1. Let $G$ be a finite non-nilpotent $\mathfrak{n n}$-group. Then $\chi\left(\mathfrak{R}_{G}\right)$ is equal to the minimum number of nilpotent subgroups of $G$ whose union is $G$. Also $\omega\left(\mathfrak{R}_{G}\right) \leq$ $\chi\left(\Re_{G}\right) \leq|G: \operatorname{nil}(G)|$.

Proof. Let $k$ be the minimum number of nilpotent subgroups of $G$ whose union is $G$ and suppose $G$ is covered by nilpotent subgroups $N_{1}, \ldots, N_{k}$. Then the vertices of $\mathfrak{R}_{G}$ in $N_{i}$ are independent. It follows that $\chi\left(\mathfrak{R}_{G}\right) \leq k$. Now assume that $\chi=$ $\chi\left(\Re_{G}\right)$. Thus there exist $\chi$ maximal independent subsets $M_{1}, \ldots, M_{\chi}$ of vertices of $\mathfrak{R}_{G}$ whose union is $G \backslash \operatorname{nil}(G)$. It follows from Proposition 3.19 that the subgroup generated by $\left\langle M_{j}, \operatorname{nil}(G)\right\rangle$ is a nilpotent subgroup of $G$, for each $j$. Clearly $G$ is covered by these $\chi$ nilpotent subgroups, so $\chi \geq k$.

It is clear that for any graph $\Gamma, \chi(\Gamma) \geq \omega(\Gamma)$. If $|G: \operatorname{nil}(G)|=m$ then $G=$ $\bigcup_{i=1}^{m} a_{i} n i l(G)$ for some $a_{1}, \ldots, a_{m} \in G$. By Lemma 3.13(i), $Z_{i}:=\left\langle a_{i}, \operatorname{nil}(G)\right\rangle$ is nilpotent for each $i$ and so $G$ is covered by $m$ nilpotent subgroups $Z_{1}, \ldots, Z_{m}$. So the first part, implies that $\chi\left(\mathfrak{R}_{G}\right) \leq|G: \operatorname{nil}(G)|$. This completes the proof.

Proposition 6.2. Let $G$ be a non-nilpotent finite $\mathfrak{n n}$-group. Then $\omega\left(\mathfrak{R}_{G}\right)=\chi\left(\mathfrak{R}_{G}\right)$. 
Proof. Assume $\omega=\omega\left(\mathfrak{R}_{G}\right)$ and $\chi=\chi\left(\mathfrak{R}_{G}\right)$. Let $x_{1}, \ldots, x_{\omega}$ be a maximal clique in $\mathfrak{R}_{G}$. Thus each $x_{i}$ is an element of $G \backslash \operatorname{nil}(G)$ and so $\operatorname{nil}_{G}\left(x_{i}\right)$ is nilpotent. It is easy to see that $G=\bigcup_{i=1}^{\omega} n i l_{G}\left(x_{i}\right)$. Now Proposition 6.1 implies that $\omega=\chi$.

Proposition 6.3. Let $q$ be a prime power number such that $q^{2} \not \equiv 1 \bmod 16$. Then

$$
\omega\left(\Re_{P S L(2, q)}\right)= \begin{cases}q^{2}+q+1 & \text { if } q>5, \\ 21 & \text { if } q=4 \text { or } 5, \\ 5 & \text { if } q=3, \\ 4 & \text { if } q=2 .\end{cases}
$$

Proof. Let $G=P S L(2, q)$, then $Z(G)=1$. By [2, Lemma 3.9], we have $n i l_{G}(a)=$ $C_{G}(a)$, for all non-trivial elements $a \in G$. Thus it follows that $\Gamma_{G} \cong \mathfrak{R}_{G}$. Thus the proof follows from [1, Lemma 4.4].

Proposition 6.4. Let $G=S z\left(2^{2 m+1}\right)$ be the Suzuki group over the field with $2^{2 m+1}$ elements, $m>0$. Let $q=2^{2 m+1}$. Then $\omega\left(\mathfrak{R}_{G}\right)=\chi\left(\mathfrak{R}_{G}\right)=q^{4}+q^{2}+1$.

Proof. The result follows from Proposition 3.7 and Proposition 6.2.

Acknowledgment. The authors would like to thank the referee for the valuable suggestions and comments.

\section{References}

[1] A. Abdollahi, S. Akbari and H. R. Maimani, Non-commuting graph of a group, J. Algebra, 298(2) (2006), 468-492.

[2] A. Abdollahi and M. Zarrin, Non-nilpotent graph of a group, Comm. Algebra, 38(12) (2010), 4390-4403.

[3] A. Azad, M. A. Iranmanesh, C. E. Praeger and P. Spiga, Abelian coverings of finite general linear groups and an application to their non-commuting graphs, J. Algebraic Combin., 34(4) (2011), 638-710.

[4] J. A. Bondy and U. S. R. Murty, Graph Theory with Applications, American Elsevier Publishing Co., Inc., New York, 1976.

[5] M. R. Darafsheh, Groups with the same non-commuting graph, Discrete Appl. Math., 157(4) (2009), 833-837.

[6] A. K. Das and D. Nongsiang, On the genus of the nilpotent graphs of finite groups, Comm. Algebra, 43(12) (2015), 5282-5290.

[7] The GAP Group, GAP - Groups, Algorithms, and Programming, Version 4.6.4, 2013 (http://www.gap-system.org). 
[8] B. Huppert and N. Blackburn, Finite Groups, III, Springer-Verlag, Berlin, 1982.

[9] B. H. Neumann, A problem of Paul Erdös on groups, J. Austral. Math. Soc. Ser. A, 21(4) (1976), 467-472.

[10] A. Yu. Ol'shanskii, Geometry of Defining Relations in Groups, Kluwer Academic Publishers Group, Dordrecht, 1991.

[11] L. Pyber, The number of pairwise noncommuting elements and the index of the centre in a finite group, J. London Math. Soc., 35(2) (1987), 287-295.

[12] D. J. S. Robinson, Finiteness Conditions and Generalized Soluble Groups, Part 2, Springer-Verlag, New York, 1972.

[13] D. J. S. Robinson, A Course in the Theory of Groups, Graduate Texts in Mathematics, 80, Springer-Verlag, New York-Berlin, 1982.

[14] D. M. Rocke, p-Groups with abelian centralizers, Proc. London Math. Soc., 30(3) (1975), 55-75.

[15] R. Schmidt, Zentralisatorverbände endlicher gruppen, Rend. Sem. Mat. Univ. Padova, 44 (1970), 97-131.

[16] R. M. Solomon and A. J. Woldar, Simple groups are characterized by their non-commuting graphs, J. Group Theory, 16(6) (2013), 793-824.

[17] V. P. Šunkov, Periodic group with almost regular involutions, Algebra i Logika, 7(1) (1968), 113-121.

[18] D. B. West, Introduction to Graph Theory (Second Edition), PHI Learning Private Limited, New Delhi, 2009.

[19] A. T. White, Graphs, Groups and Surfaces, North-Holland Mathematics Studies, 8, American Elsevier Publishing Co., Inc., New York, 1973.

[20] C. Wickham, Classification of rings with genus one zero-divisor graphs, Comm. Algebra, 36(2) (2008), 325-345.

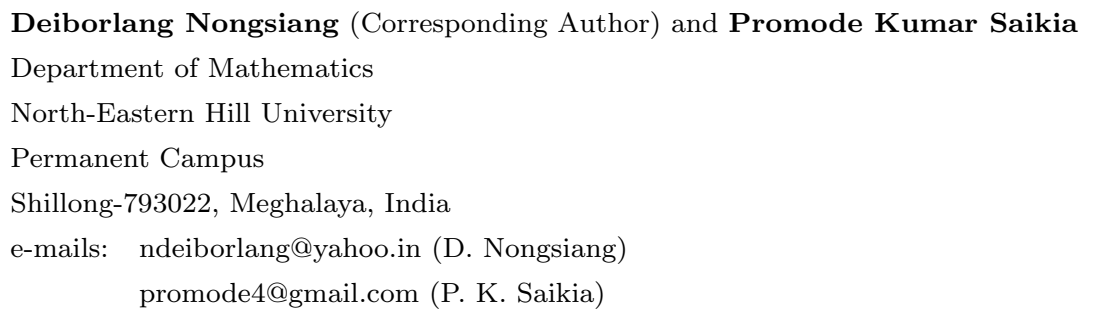

